\title{
Mobile computers in a Community NHS Trust. Is this a relevant context and environment for their use?
}

\author{
Barbara McManus \\ Dept of Computing, University of Central Lancashire, Preston, Lancs, PR1 2HE. Tel: 01772893288. \\ Email: b.mcmanus@uclan.ac.uk
}

\begin{abstract}
This paper describes a case study in which mobile computers were used by a UK NHS Trust. The intention was to give clinicians access to up to date patient data enabling them to be more clinically effective in their jobs. Since the majority of their work was performed in the community, it was perceived that the use of palmtop computers would give the flexibility needed to allow this clinical effectiveness to occur. This paper will attempt to demonstrate how this was in reality and point to various factors that had not been considered prior to the introduction of the system. Had these factors been considered, a different system would have been designed with a better fit between the needs of the user, the organisation and their work environment.
\end{abstract}

\section{BACKGROUND}

In 1992 the first NHS IM\&T (Information Management and Technology) strategy (DoH, 1992) was published. Whilst this has been heralded as an international leader, it has been criticised for being too management oriented (DoH, 1998). In 1997 following a change of government the second wave of NHS reforms were introduced through the 1997 White Paper 'The New NHS' (DoH, 1997). This was followed by the new IM\&T strategy in 1998 (DoH, 1998), which promised a more clinical focus. Under the new NHS, local trusts are under pressure to reduce administrative costs. IT has been offered as a route to achieve this. In this study, we shall consider the introduction of new IT into a Community Health Trust within this context and examine the issues arising.

Since the 1980s the UK NHS (National Health Service) has gradually adopted IT (Information Technology). However in spite of significant investment and a large amount of technology, the quality of information systems, when viewed in terms of their ability to support the delivery of health care, remains patchy.

The first wave of NHS reforms was based around the 1987 White Paper (DoH, 1987). This established the need for management information based around access to health care; quantity of care delivered and costs associated with procedures. Major reforms followed in Primary and Community Care, notably the 1989 White Paper (DoH, 1989) which required doctors to undertake activities such as health promotion which were information intensive. This led to a major growth in computerisation, for example in the mid 1980s one quarter of family doctors had computer systems. By 1996 this figure had risen to 96\% (NHSE, 1997).

\section{CONTEXT OF THE PROJECT}

To position this case study within a context, it is appropriate to describe the Community NHS Trust within which it took place. The Trust consists of around 400 nurses and PAMs (Professionals Allied to Medicine), serving a largely urban new town area. It has a varied social profile and a limited racial profile. There is a traditional NHS culture within the Trust and it could be declared that like many Trusts in similar positions, the NHS reforms had had a limited impact within the Trust. The clinical staff within the Trust had limited exposure to Information Technology, and the IT department is small for an organisation of this size, when compared to commercial equivalents.

One of the main strengths of the Trust is in its committed workforce who showed a high level of professionalism in the course of its everyday work. It does however suffer from a lack of both IT infrastructure as well as being deficient in project planning expertise.

The project did offer some opportunities to the Trust; namely the use of new technology and the transferable skills that it would bring into the workforce, together with the promise of reduced administration costs. However there were some threats being felt within the Trust. The Trust Management was worried by the latest Government initiative of Primary Care Groups (PCGs). The clinicians were worried by the potential reduction in the time available to them to spend with patients and both groups were apprehensive at the reduction in administration staff and the further implications that indicated. 


\section{REASONS FOR THE PROJECT'S LIMITED SUCCESS}

The project has had limited success; it is being used by the clinicians but, with the exception of the early adopters whose findings led to changes being made to the software, early indications show that the clinicians feel disenfranchised which leads them to negative feelings towards the project. This indicates that users whose opinions have been sought are more likely to accept the introduction of new technology. Robertson et al (Robertson, 1996) saw this with the introduction of palmtops into secondary schools for teachers' use. They noted that the staff needed to have a sense of control over the introduction of new technology, saying that 'If teachers are expected to embrace the new technologies then they should have a say in how and when they are introduced.' If the clinicians had been involved they would have been more accepting of the project. The clinicians would have been able to point out some of the limitations of the system; namely the problem of assigning each clinician to only one download site and the ten minute download period highlighted in section 3.5 of this paper.

Physical limitations of the hardware and software emphasised the user's physical limitations, did not compensate for them. The software usability aspects are not considered here since they can be found in a partner paper (McManus, 1999).

\subsection{Contextual and Environmental Limitations}

The hardware limitations had not been considered although some of the clinicians had seen the hardware and along with other palmtops, tried it out, this evaluation method took no account of the environment in which the hardware would be used (i.e. in varying conditions and for long periods of time). Nor did it consider the problems associated with the mechanism for maintaining up to date records on the clients. Since the clinicians worked mainly in the community, travelling round client's homes or to various clinics in the area making the upload/download process necessary to maintain reasonably up to date data, very difficult. Even charging the batteries was not straightforward. Many of the clinics had few power sockets in the clinicians' offices making recharging difficult. It was soon discovered that if the clinicians were on holiday, they had to negotiate for someone else to charge their machine otherwise on their return they would be greeted with a 'battery low message' frequently popping up to indicate the need to change the back up battery. This interfered with their thought processes and became yet another source of annoyance to them.

\subsection{Physical limitations}

The physical design size of the mobile computer was a problem to many. The screens were far too small for the amount of information necessary to the clinicians to enable them to do their job efficiently. Although enlarging the font may have made it easier to see the text, it could have detracted from the user's model of the system. Many users had problems with the limited contrast on the machine. Frequently, when they were unable to see the screen and its contents, they made the text darker (thereby decreasing the contrast) making any 'greyed out' options appear available to the users. This, combined with an inconsistently designed interface caused a lot of confusion. The keyboard was designed to follow a standard QWERTY layout. Trained typists found this difficult because their knowledge of the position of the letters on the keyboard had dropped to a subconscious level. This meant that finding the letter was associated closely with the ability to type on a standard sized keyboard. Since this was not standard, the typing training was of little use; they had to adopt the 'hunt and peck' mechanism in the same way as the non-typists although they were slightly faster than the untrained typists were.

An additional problem was the physical size and design of the keys on the keyboard. They were small with no chamfered sides and a gap around the key. Many users had difficulties typing, moving in favour of using the blunt end of the screen pointer rather than their finger, finding that this caused them to make fewer mistakes. The greatest problem was due to the lack of consideration for the way in which palmtops could be well used in its environment. Focus had been given to the mobile aspects of computing without full consideration of the implications associated with it: namely that of data integrity. The aim of the project was to make the clinicians clinically effective, but to be clinically effective they needed immediate access to complete, reliable, up to date data. Implied collaboration was essential for this to be the case; each clinician had to be responsible for the data maintenance. They had to ensure that the data entered on their palmtop was correct, that they downloaded their changed data to the host so others had the benefit of their knowledge and uploaded from the host the data changes made by their colleagues. The full implications of this had not been considered. One of the main limitations is that mobile computers frequently require access to data on a host due to the memory limitations. This was noted by Kristoffersen and Ljungberg where they point out that 'in order to make the mobile computer support mobile work it has to be turned into a desktop computer by connecting it to the stationary network and funnel its functionality through a hosting PC'. 


\subsection{Resource Limitations}

The palmtop has limited data capacity yet many of the clinicians have large client caseloads or caseloads which change on a frequent basis. This has meant that the clinicians often don't have on their palmtops, details of that client. Because they must keep a record of their work on the palmtop, they must enter all the details pertaining to that client even though those personal details are frequently to be found on the host computer. On the paperbased systems (that the computer one replaces), this was not a problem since they wrote down the client's unique number together with date, location, time taken and action taken. On the computer system, if that client is not on the clinician's palmtop they must enter personal details, GP details on the referral followed by the contact details as per paper based. This can take around 15 minutes per client.

\subsection{Model Mismatch?}

Since the computer version forces the clinician to enter in all the details and those details are transferred to the host it is unclear whether that record should be considered as a New Record or as a record to update one which is already in place. In other words, if the clinician got some details wrong, what effect would this have on the host data? The author is aware of one problem area. In the personal part of the record if the clinician omitted the postcode of the palmtop version, this overwrote the host computer's record, replacing a valid field with a blank field. This was realised and the software changed to not overwrite the postcode. However this means that an address and postcode don't match. In itself this does not appear to be a problem (note that at present there is no software on the system to locate the PC according to address or vice versa) until it is realised that the various host systems are being merged and another of the systems that accesses this one bases its costing on the client's postcode.

\subsection{Synchronisation to maintain up to date data on palmtop}

The clinicians know the importance of the upload/download procedure to maintain up to date data but the mechanism provided to allow this to take place interferes with their main work. To perform the upload/download they must return to their specified download site to link up to their specified partner PC. Since the majority of them work in the community or at a variety of clinics, this has to be timed to minimise the disruption to their work. Consequently this results in large numbers of users needing to use the PC at the beginning or the end of the day. By the time the clinician has set up the PC and the palmtop for downloading and done the download, at least 10 minutes has passed. When this period is multiplied by the number of clinicians assigned to the site, a bottleneck is seen to occur. As a result many clinicians chosen not to upload/download on a daily basis with the consequences that neither they nor their colleagues have access to the most up to date data. This need to overcome resource limitations and the need for frequent synchronisation was highlighted as an area for discussion by Colleen Page (Page, 1998) in her position paper for the Workshop on Handheld CSCW at CSCW 98 in Seattle. Since her position is that of a Usability Engineer working for Microsoft Corporation, this perhaps indicates an improvement in the Windows CE Services mechanism for the synchronisation of data between mobile and host computers. If this were to be the case it would vastly improve one of the greatest limitations with systems such as the one described above.

\subsection{One client seen by multiple clinical service groups}

Further limitation can be seen when a client is seen by more than one member of the same service group; e.g. Community Psychiatric Nurses (CPNs) form part of the same service group as the Alcohol and Drugs Team. If a client is seen by both CPNs and the Drugs Team they cannot appear on the palmtops of both groups. One has the client on their palmtop and the other must enter all the details (as if for a New Client) each time they see the client. This is not only wasteful of resources (time) but also extremely frustrating to the clinicians.

\section{DISCUSSION OF PROBLEMS}

The problems highlighted above point to a number of oversights on the part of the project team. The users and their needs and particularly their environment have not been well considered. According to Johnson (Johnson, 1998) 'Research in Human Computer Interaction has recently begun to acknowledge the importance of the users' context and environment when designing interactive systems. The designers did not consider the clinicians' working environment and in particular did not consider their mode of working and its implications. Johnson also highlights (Johnson, 1998) that success will only be realised if 'designers have a clear idea of the requirements that mobile systems must satisfy'. This case study is also proof of a third area highlighted in the workshop, that 'even if it is possible to identify user requirements for mobile computing devices, it is far from clear whether we have appropriate devices to satisfy their needs'. Not only are the devices themselves a problem, but also the mechanism by which these devices are made usable to the end user. The method of and limitations caused by the synchronisation of the devices with their host computer limits the functionality since it is this that allows the user 
to be effective. So we see in this case study that although the palmtops allowed the clinicians to enter data and have limited access to client data remotely, The act of synchronisation was a deterrent to the maintenance of up to date data. The consequence of this and the limited resource (in terms of memory) meant that the clinicians are not clinically effective and unless the design changes fundamentally are unlikely to be so in the foreseeable future.

\section{REFERENCES}

Johnson (1998), First Workshop on Human Computer Interaction with Mobile Devices, 21-23 May 1998, http://www.dcs.gla.ac.uk/ johnston/papers/mobile/

Kristoffersen, S and Ljungberg, F (1998), Workshop on Handheld CSCW at CSCA '98, Seattle

Page, C (1998), Workshop on Handheld CSCW at CSCA '98, Seattle

Robertson, S.I., Calder, J., Fung, P., Jones, A., O'Shea, T., Lambrechts, G., (1996), Pupils, teachers and Palmtop computers in Journal of Computer Assisted Learning (1996) 12, 194-204 